\title{
ANÁLISE DA PREVISÃo PLUVIOMÉTRICA A CURTÍSSIMO PRAZO NA BACIA HIDROLÓGICA DO RIO PARAÍBA DO MEIO UTILIZANDO O MODELO WRF
}

\author{
SANTOS, André Gonçalo dos - andregs9@yahoo.com.br \\ Doutorando em Engenharia de Recursos Hídricos pela Universidade Federal do Ceará
}

\begin{abstract}
CAMPOS, José Nilson Beserra
Professor, Doutor do Departamento de Engenharia Hidráulica e Ambiental da Universidade Federal do Ceará
\end{abstract}

SILVA JUNIOR, Rosiberto Salustiano da

Professor, Doutor do Instituto de Ciências Atmosférica da Universidade Federal de Alagoas.

\begin{abstract}
RESUMO: $O$ presente estudo têm por objetivo analisar como os diferentes esquemas físicos e suas combinações influenciam nas previsões de chuva a curtíssimo prazo no vale do rio Paraíba do Meio. Para este propósito, foi utilizada uma análise estatística para avaliar os resultados de precipitações obtidas em cada uma das nove combinações diferentes de esquemas microfísicos e convectivos, usados no modelo de mesoescala WRF, em um horizonte de 120 horas. A aquisição dos dados para validação dos resultados foi obtida junto a Agência Nacional de Águas. A pesquisa mostrou que o WRF têm um tendência em subestimar as precipitações pluviométricas de curtíssimo prazo na bacia do rio Paraíba do Meio. E oferecer uma maior destreza nas previsões usando a combinação entre os esquemas de MPL e G3D.
\end{abstract}

Palavra Chave: WRF, Previsões, Esquemas paramétricos.

VERIFICATION OF VERY SHORT TERM RAINFALL FORECAST OVER THE BASIN OF THE PARAÍBA DO MEIO RIVER USING DIFFERENT COMBINATIONS PARAMETRIC IN THE WRF

ABSTRACT: This study objective is analyze how the different physical schemes and their combinations influence forecasts of rain in the short term on the River valley Paraíba do Meio. For this purpose, was used a statistical analysis to evaluate the results of precipitations obtained at each of nine different combinations of microphysics and convective schemes, used in the WRF mesoscale model in a horizon of 120 hours. The acquisition of data for validation of the results was obtained at the National Water Agency. Research has shown that the WRF have a tendency in underestimate the rainfall the short term in the Paraíba do Meio River basin. And it offers a greater dexterity in the combination of forecasts using schemes MPL and G3D.

Keyword: WRF, Forecasts, parametric schemes.

\section{INTRODUÇÃO}

A bacia do rio Paraíba do Meio estar localizada entre os estados de Pernambuco e Alagoas, e é distribuída em 21 municípios entre os dois estados, sendo oito em Pernambuco e treze em Alagoas. Esta região como muitas outras no Brasil, tem apresentado problemas nos seus planejamentos de ações de contingências elaboradas com o propósito de minimizar os impactos de eventos extremos de precipitações. Problemas como estes são reflexos das informações pluviométricas com pouca consistente, baseadas em modelos numéricos de tempo.

As previsões pluviométricas a curtíssimo prazo são fundamentadas em modelos numéricos meteorológicos de tempo, que fornece informações importantes para as ações de contingência, permitindo a possibilidade de prever eventos extremos com antecedência que permite a sociedade tomar decisões que reduza os impactos de tais eventos.

Um dos modelos numéricos mais difundidos no Brasil e no mundo é o WRF, utilizado não apenas na previsão temporal de precipitação pluviométrica mais para diversas outras componentes atmosféricas (MACEDO et al., 2011; RAMOS et al., 2013; MOLLMANN JUNIOR et al., 2013; OLIVEIRA, 2006). A qualidade das previsões depende do ajuste paramétrico de cada modelo (ARGÜESO et al., 2011). O WRF disponibiliza vários esquemas paramétricos que ajudam a obter uma melhor adequação paramétrica para cada região e consequentemente um melhor resultado. Dessa forma o presente estudo objetiva analisar como os diferentes esquemas físicos e suas combinações influenciam nas previsões de chuva a curtíssimo prazo 
no vale do rio Paraíba do Meio, no sentido de demonstrar a eficiência do modelo atmosférico em fazer a previsão.

\section{METODOLOGIA E DADOS}

\subsection{Modelo WRF}

O modelo de mesoescala "Weather Research and Forecasting" (WRF) versão "Advanced Research WRF" (ARW) é de acesso livre ao público sendo necessário conhecimento básicos de informática para sua instalação, e é resultado de uma parceria conjunta entre uma série de instituições e agências governamentais, entre elas: o Centro Nacional de Pesquisas Atmosféricas (NCAR), a Administração Nacional de Oceanos e Atmosfera (NOAA) e o Centro Nacional de Previsão do Meio Ambiente (NCEP) (SKAMAROCK et al., 2005). Estas mesmas instituições mantem um esforço conjunto e contínuo em busca do seu aprimoramento. $O$ modelo apresenta uma diversidade de opções de esquemas dinâmicos e parametrizações físicas, tornando-o adequado para a previsão numérica e simulação de clima (SKAMAROCK et al., 2008).

O modelo WRF utiliza em sua estrutura o sistema de coordenadas verticais denominado eta $(\eta)$. Nesse sistema cada nível é representado por um valor médio para a parcela entre as duas superfícies, discreto por $\eta$. Esse valor médio é expresso pela equação (1).

$$
\eta=\frac{\left(p_{h}-p_{h t}\right)}{\mu}
$$

Onde $\mu=p_{h s}-p_{h t}, p_{h}$ é a componente hidrostática da pressão, $p_{h t}$ é o valor no topo e $p_{h s} 0$ valor na superfície.

\subsection{Dados}

Como condições iniciais e de contorno, foram utilizados os dados do modelo atmosférico global "Global Forecast System" (GFS). Esses dados são fornecidos pelo "National Centers for Environmental Prediction" (NCEP) em quatro rodadas diárias de 6 em 6 horas com início às 00 UTC, e com resoluções espaciais de 0,5, 1 e 2,5 graus de latitude e longitude por uma resolução temporal de 3 horas, e horizonte de até 384 horas. As simulações com o modelo WRF foram realizadas utilizando os dados da rodada das 00 UTC, com resolução espacial de $1,0^{\circ} \times 1,0^{\circ}$ (aproximadamente $111 \mathrm{~km}$ ).

Por outro lada, os dados utilizados na validação, foram fornecidos pela Agência Nacional de águas (ANA) disponibilizados nos dados hidrológicos em tempo real. Esses são coletados por um sistema denominado Telemetria nas estações automáticas, com a utilização de Plataformas de Coletas de Dados (PCDs) na qual suas transmissões são efetuadas pelos satélites brasileiros (SCD e CBERS). Duas séries foram extraídas de duas estações fluviométrica, uma localizada na cidade de Atalaia $\left(-9030^{\prime} 24,12^{\prime \prime}\right.$ e $\left.-36^{\circ} 1^{\prime} 22,08^{\prime \prime}\right)$ e a outra em Viçosa $\left(-92^{\circ} 22^{\prime} 45,12^{\prime \prime}\right.$ e $36^{\circ} 14^{\prime} 57,12^{\prime \prime}$ ) ambas em Alagoas, de um período de 5 dias compreendidos entre os dias 27 a 31 de Julho de 2011.

\subsection{Metodologia}

O WRF foi configurado com dois domínios aninhados, o primeiro (D1) (Figura 1) envolvendo toda a região Nordeste (NE), com resoluções espaciais de $20 \mathrm{~km}$ e o segundo (D2) cobrindo toda a região da bacia do rio Paraíba do Meio localizada entre o estado de Pernambuco e Alagoas e com resoluções espaciais de $5 \mathrm{~km}$, apresentados na Figura 1, e com uma configuração de 31 níveis sigma na vertical. A simulação foi feita para um horizonte temporal de 120 horas, tendo início às 00 UTC do dia 27 de Julho de 2011 e termino às 00 UTC do dia 31 de Julho de 2011. O processamento foi feito com o armazenamento dos resultados em intervalos de 1 hora para ambos domínios de integração. 


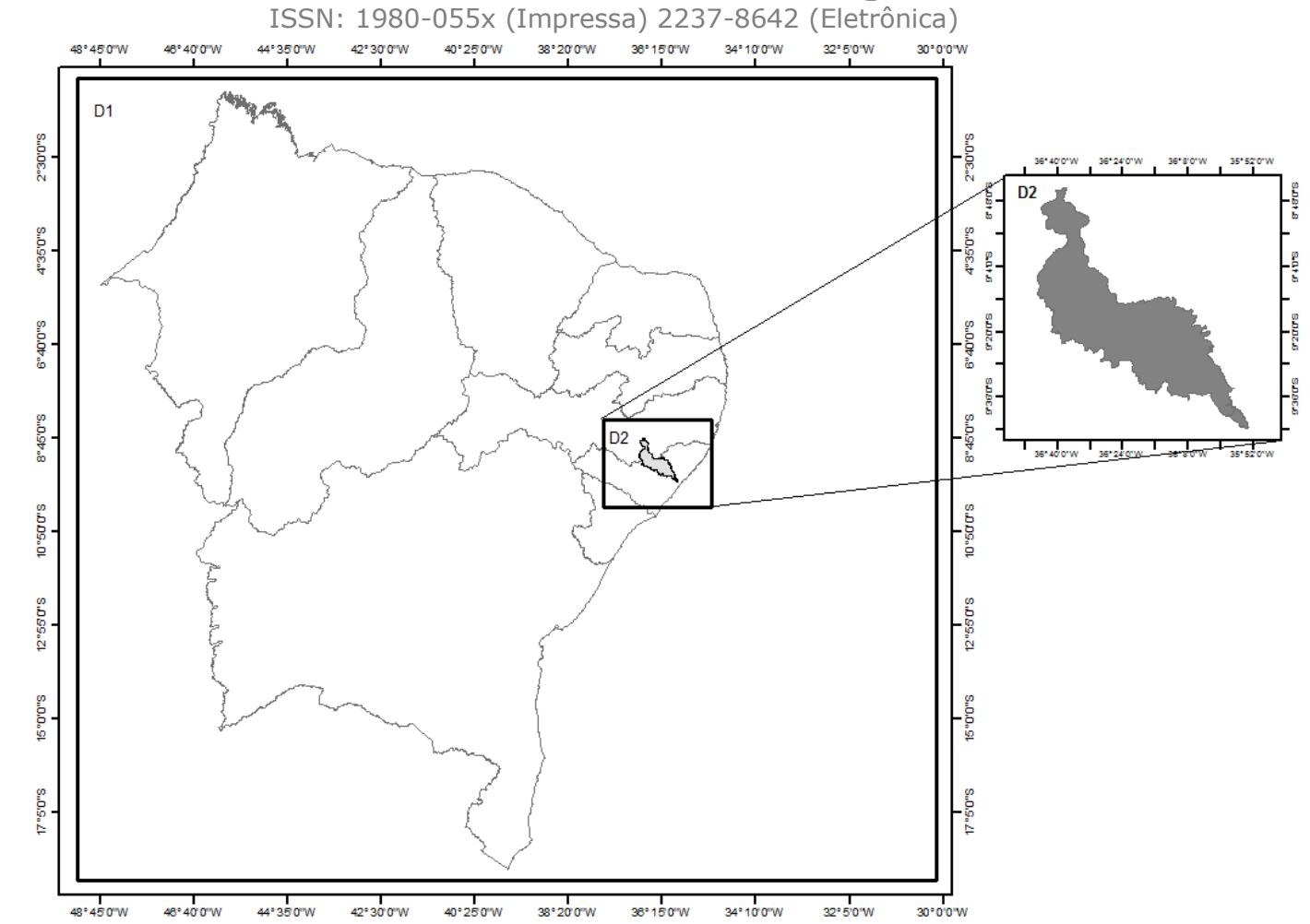

Figura 1: Representação dos domínios configurados no modelo WRF.

\begin{tabular}{ll}
\hline \hline Esquema Físico & Notação \\
\hline Kessler - Microfísica & MPK \\
Lin et al. - Microfísica & MPL \\
Thompson - Microfísica & MPT \\
Kain-Fritsch - Convecção & KF \\
Betts-Miller-Janjić - Convecção & BMJ \\
Grell 3D - Convecção & G3D \\
\hline \hline
\end{tabular}

Tabela 1: Notação usada para diferentes sistemas físicos no presente estudo.

Foi criada uma matriz de nove variantes usando diferentes combinações de microfísicas e convecção do modelo WRF (Tabela 2), com o esquema de Kessler (1969), Lin et al. (1983) e Thompson (2004) por três esquemas de convecção, os esquemas de Kain-Fritsch (1993), Betts-Miller-Janjić (Betts e Miller 1986; Janjic 1994) e Grell 3D que é uma versão aperfeiçoada do sistema de Grell-Devenyi (GRELL e DEVENYI, 2002) que por sua vez pode ser utilizado em alta resolução. Para os outros parâmetros no WRF, fixamos os esquemas RRTMG (MLAWER et al., 1997) para radiação de onda longa; o esquema de Goddard para radiação de onda curta; o MM5 similaridade (PAULSON, 1970; DYER e HICKS, 1970; WEBB, 1970) para camada superfície; o Noah-LSM (CHEN e DUBHIA, 2001) para processos de superfície e ACM2 PBL (PLEIM, 2007) para camada limite planetária.

\begin{tabular}{cccc}
\hline \hline & $\mathrm{KF}$ & $\mathrm{BMJ}$ & $\mathrm{G} 3 \mathrm{D}$ \\
\hline $\mathrm{MPK}$ & $\mathrm{MPK}+\mathrm{KF}$ & $\mathrm{MPK}+\mathrm{BMJ}$ & $\mathrm{MPK}+\mathrm{G} 3 \mathrm{D}$ \\
$\mathrm{MPL}$ & $\mathrm{MPL}+\mathrm{KF}$ & $\mathrm{MPL}+\mathrm{BMJ}$ & $\mathrm{MPL}+\mathrm{G} 3 \mathrm{D}$ \\
$\mathrm{MPT}$ & $\mathrm{MPT}+\mathrm{KF}$ & $\mathrm{MPT}+\mathrm{BMJ}$ & $\mathrm{MPT}+\mathrm{G} 3 \mathrm{D}$ \\
\hline \hline
\end{tabular}

Tabela 2: Possíveis combinações de parametrizes físicas utilizando três (3) esquemas de microfísicas por três (3) de convecção no modelo WRF.

Para avaliar as precipitações previstas por cada combinação, foram utilizados índices estatísticos, como: o erro médio (VIÉS), que indica a direção do erro (um Viés positivo indica 
superestimação e um negativo subestimação dos valores observados), a Raiz do Erro Quadrático Médio (REQM), que mede a amplitude do erro (o valor zero indica uma previsão ideal) e por fim o coeficiente de correlação de Pearson ( $r$ ), que mede o grau de relação linear entre duas variáveis quantitativas. $O$ valor de $r$ apresenta uma variação entre -1 e 1 . $O$ sinal (positivo ou negativo) e o valor sugere a direção e a força deste relacionamento, enquanto o um 0 (zero) indica a ausência de correlação.

Os valores destes índices estatísticos VIÉS (2), REQM (3) e r (4) são calculados pelas seguintes equações:

$$
\begin{gathered}
V I E ́ S=\frac{1}{N} \sum_{i=1}^{N}\left(P_{i}-P_{O b s}\right) \\
R E Q M=\sqrt{\left(N^{-1} \sum_{i=1}^{N}\left(P_{i}-P_{O b S}\right)^{2}\right)} \\
r=\frac{\sum_{i=1}^{N}\left(x_{i}-\bar{x}\right)\left(y_{i}-\bar{y}\right)}{\sqrt{\left(\sum_{i=1}^{N}\left(x_{i}-\bar{x}\right)^{2}\right)\left(\sum_{i=1}^{N}\left(\left(y_{i}-\bar{y}\right)^{2}\right)\right.}}
\end{gathered}
$$

Onde $P_{i}$ e $x_{i}$ são as precipitações previstas, $P_{o b s}$ e $\bar{y}$ são a precipitações observadas na superfície e $\mathrm{N}$ é o número de dados analisados.

Já na avaliação da habilidade preditiva do modelo WRF, foi utilizado o índice de Heidke Skill Score (HSS). O HSS mede a habilidade da previsão de uma variável em relação a um padrão particular, tal como a previsão feita ao acaso, ou seja, ele elimina a influência de previsões que estariam corretas por acaso. Segundo Conner e Grant (1998), o HSS indica o grau de concordância entre dois pressupostos binários como chover ou não chover. O HSS é calculado a partir de uma tabela de contingência de $2 \times 2$, tal como definido pela tabela 3 (DOSWELL et al.,1990; BARNSTON, 1992; LEE E PASSNER, 1993; WILKS, 1995). O HSS pode variar entre -1 a 1 , com 1 indicando uma habilidade perfeita, zero, indicando nenhuma habilidade ou uma previsão equivalente a uma previsão de referência, que neste caso significa acaso, e -1 , indicando desempenho pior do que um esquema aleatório. E com base nas definições dos elementos da tabela 3, o HSS é calculado pela equação 5 .

\begin{tabular}{cccc}
\hline \hline \multirow{2}{*}{ Observação } & \multicolumn{3}{c}{ Previsão } \\
& Choveu & Não Choveu & Total \\
\hline Choveu & $\mathrm{X}$ & $\mathrm{y}$ & $\mathrm{x}+\mathrm{y}$ \\
Não Choveu & $\mathrm{z}$ & $\mathrm{w}$ & $\mathrm{z}+\mathrm{w}$ \\
Total & $\mathrm{x}+\mathrm{z}$ & $\mathrm{y}+\mathrm{w}$ & $\mathrm{t}$ \\
\hline \hline
\end{tabular}

Tabela 3: Esquema de contingência.

Onde: $x$ - Número de eventos de previsão de ocorrência de precipitação que foram realmente observadas; y - Número de eventos de previsão de ocorrência de precipitação que não houve observação de chuvas; z - Número de eventos de previsão de não ocorrência de precipitação que ocorreu observação de chuvas; w - Número de eventos de previsão de não ocorrência de precipitação que realmente não houve observação de chuvas e $T($ Total $)=(x+z)+(y+w)=$ $(x+y)+(z+w)$.

$$
H S S=\frac{2(x w-y z)}{[(x+z) \cdot(z+w)+(x+y) \cdot(y+w)]}
$$




\section{RESULTADOS}

Os resultados apresentados neste estudo referem-se às simulações de alta resolução ( $5 \mathrm{~km}$ de grade) no D2.

A tabela 4 traz a estatística para cada uma das nove combinações dos esquemas paramétricos convectivos e microfísicas em Atalaia. A análise estatística evidenciou que o modelo WRF-ARW subestimou as previsões em todas as combinações com uma magnitude do erro entre as precipitações previstas e a observadas próximos a zero. O VIÉS indicou a microfísica de Thompson et al. (2004) e a parametrização convectiva de Kain-Fritsch (1993) como os parâmetros que gerou menor valor de subestimação $(-0,07)$, classificado como boa segundo Moriasi et al. (2007). O REQM também destacou os dois esquemas como os que apresentou o menor erro na intensidade entre o conjunto de dados observados e o previsto. As correlações entre os conjunto de dados previstos e observados foram considerados bons na maioria das rodadas, contudo, o esquema de MPT e KL apresentou um $r$ de $68 \%$ entre os valores observados e previstos, que é considerado uma correlação forte segundo Callegari-Jacques (2003) e Lira (2004).

\begin{tabular}{cccc}
\hline \hline COMBINAÇÃO & $r$ & VIÉS & REQM \\
\hline MPK e KF & 0,55 & $-0,01$ & 1,82 \\
MPK e BMJ & 0,31 & $-0,84$ & 2,17 \\
MPK e G3D & 0,53 & $-0,55$ & 1,87 \\
MPL e KF & 0,63 & 0,07 & 1,66 \\
MPL e BMJ & 0,44 & $-0,81$ & 2,08 \\
MPL e G3D & 0,46 & $-0,27$ & 2,03 \\
MPT e KF & 0,68 & $-0,07$ & 1,55 \\
MPT e BMJ & 0,38 & $-0,82$ & 2,12 \\
MPT e G3D & 0,61 & $-0,28$ & 1,69 \\
\hline \hline
\end{tabular}

Tabela 4: Estatística relacionada a cada uma das combinação de microfísica e convecção simulada pelo modelo WRF-ARW para o posto pluviométrico de Atalaia/AL.

A precipitação prevista pelo modelo WRF-ARW usando os esquemas de MPT e KF foram comparadas aos dados pluviométricos observados (Figura 2). Os dados reais são da mesma localidade para onde foram feitas as previsões (Cidade de Atalaia). A análise constatou que o modelo conseguiu visualizar as precipitações em todos os instantes onde se obteve chuva real, contudo, a magnitude das chuvas previstas foram diferentes como apontadas pelo Viés e o REQM (Tabela 4). Uma possível explicação para essa diferença, pode estar relacionada a distância entre o ponto de grade de onde foi extraída a precipitação prevista e o posto pluviométrico de onde foi coletado os dados para validação, que é de aproximadamente de 2 quilometro. Segundo Yuan et al. (2009) e Argüeso et al., 2011, o modelo WRF tem seu melhor desempenho em previsões de escalas maiores como médio e longo prazo. Contudo, a precipitação acumulada prevista dos cinco dias analisados, correspondeu a $94 \%$ da observada, valor altamente significativo. 


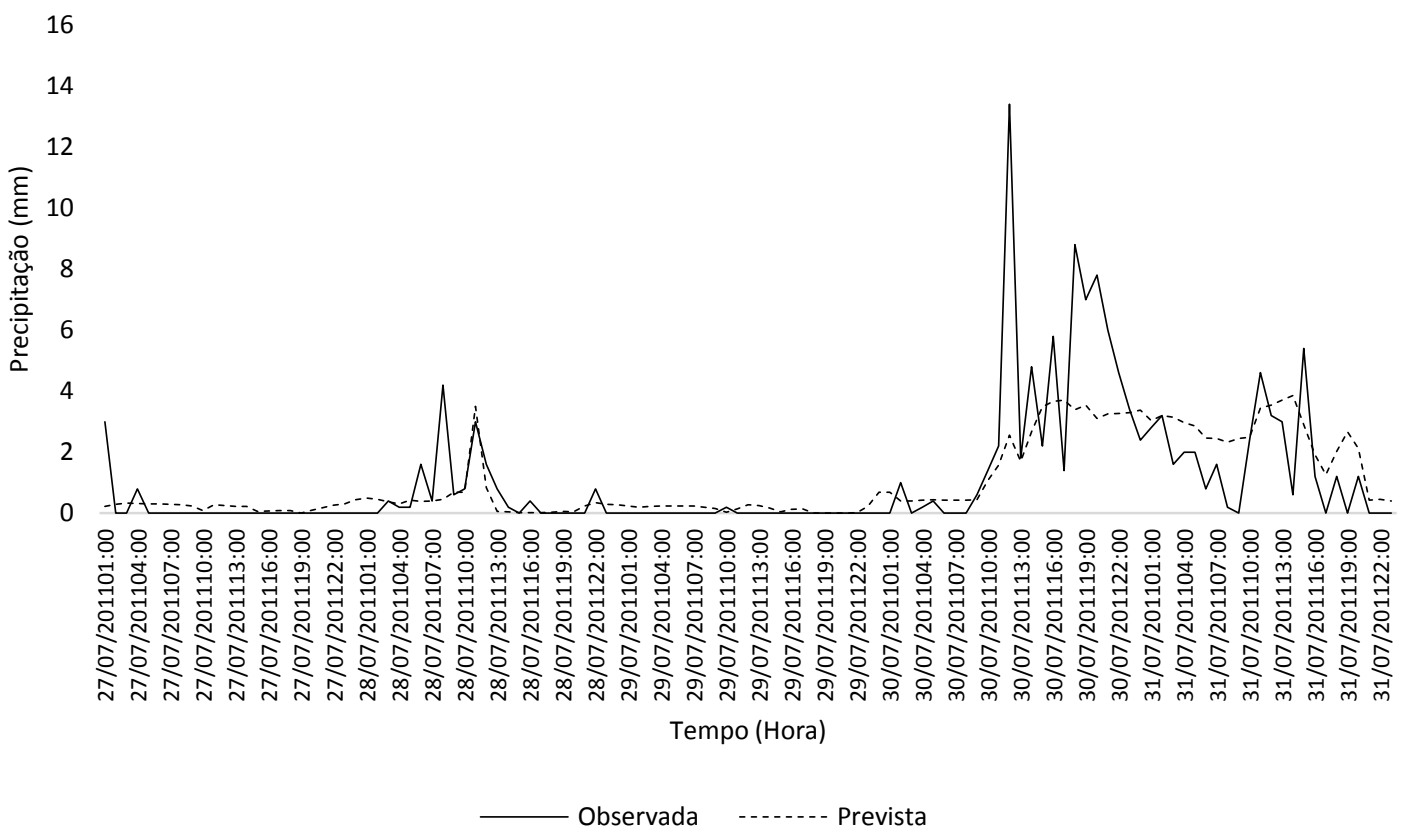

Figura 2: Comparação entre a precipitação observada e a simulada, utilizando o esquema de microfísica de Thompson e o esquema convectivo de Kain-Fritsch, para o posto pluviométrico de Atalaia/AL.

No segundo ponto analisado que corresponde a cidade de Viçosa, foi obtido resultado diferente dos alcançados em Atalaia (Tabela 5). Apesar da MPL e o esquema convectivo de KF apresentarem um melhor coeficiente de correção $(0,46)$ comparado as outras combinações, as chuvas acumuladas no período de análise não ultrapassou os $13 \%$ da observada, indicando uma elevada subestimação das chuvas previstas. Já o esquema de MPT e a parametrização convectiva G3D proporcionou um melhor resultado, apontado pela estatística, com correlação igual $40 \%$, considerado um valor moderada segundo Callegari-Jacques (2003), e um Viés igual a $-0,42$ e REQM igual a 1,89. O modelo subestimou o resultado previsto em todas as rodadas, com magnitude não muito diferente as de Atalaia, utilizando os esquemas de MPT e KF. O esquema G3D foi o que mais se adequou a região da cidade de Viçosa como indicado pela estatística. E uma possível explicação para este fato, pode estar nas condições topográficas e cobertura do solo na região, visto que a cidade de Viçosa está localizada em uma área com relevos mais acentuados e com uma maior cobertura vegetal quando comparada a cidade de Atalaia.

\begin{tabular}{cccc}
\hline \hline COMBINAÇÃO & $r$ & BIAS & REQM \\
\hline MPK e KF & 0,42 & $-0,97$ & 2,05 \\
MPK e BMJ & 0,36 & $-0,93$ & 2,04 \\
MPK e G3D & 0,29 & $-0,61$ & 2,80 \\
MPL e KF & 0,46 & $-1,02$ & 2,08 \\
MPL e BMJ & 0,42 & $-0,95$ & 2,03 \\
MPL e G3D & 0,42 & $-0,50$ & 2,19 \\
MPT e KF & 0,21 & $-0,99$ & 2,14 \\
MPT e BMJ & 0,20 & $-0,95$ & 2,14 \\
MPT e G3D & 0,40 & $-0,42$ & 1,89 \\
\hline \hline
\end{tabular}

Tabela 5: Estatística relacionada a cada uma das combinação de microfísica e convecção simulada pelo modelo WRF-ARW para o posto pluviométrico de Viçosa/AL. 
Na validação da precipitação prevista para Viçosa (Figura 3), foi utilizado dados observados da região. Na análise foi possível observar que o modelo WRF-ARW simulou aparentemente a precipitação no período analisado, e, previu apenas $64 \%$ das chuvas acumuladas. Essa diferença no valor acumulado previsto, poderia estar relacionado a não exatidão entre o ponto de grade e a estação pluviométrica usada na validação, que distam uma da outra aproximadamente 1300 metros. Esses resultados evidenciam apenas a estatística existente entre os conjuntos de dados previstos e o observados, e não à análise da habilidade do WRF.

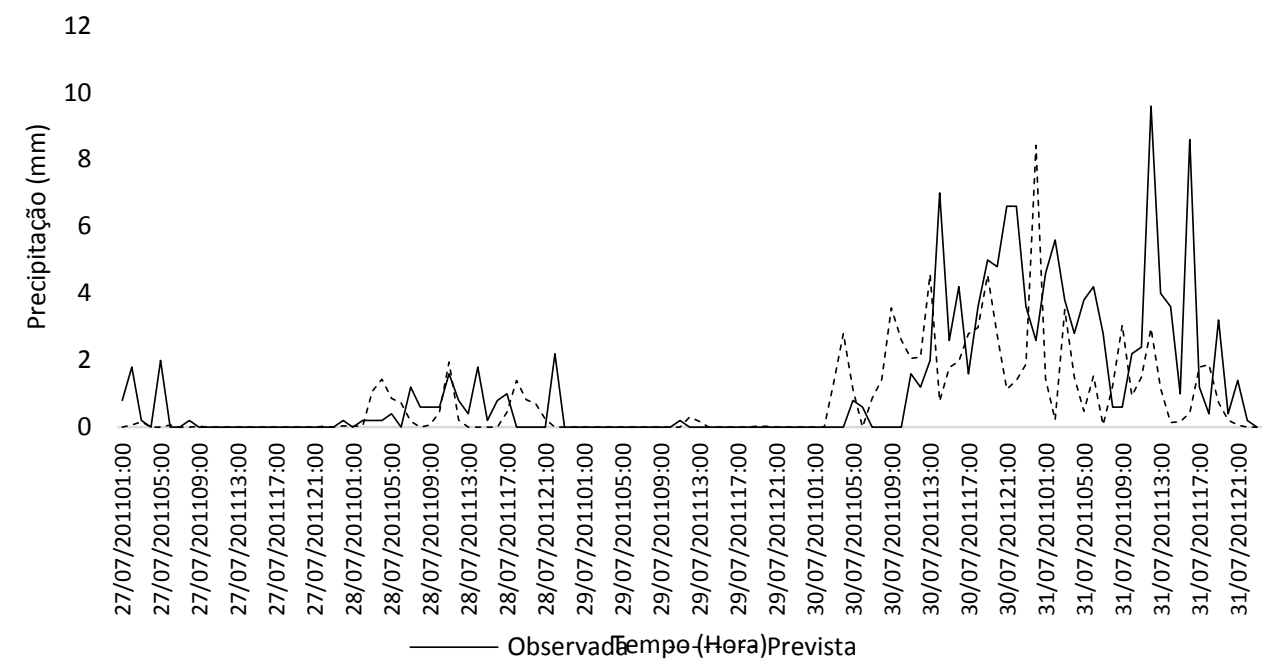

Figura 3: Comparação entre a precipitação observada e a simulada, utilizando o esquema de microfísica de Thompson e o esquema convectivo de Grell 3D, para o posto pluviométrico de Viçosa/AL.

A figura 4 mostra a análise da destreza do modelo WRF em um horizonte de 120 horas na previsão de chuva, feita para cada uma das nove combinações discutidas anteriormente nos dois pontos escolhidos (Atalaia e Viçosa). O índice HSS apresentou valores que informa a capacidade preditiva do WRF em cada uma das rodadas. O modelo proporcionou bons resultados preditivos em quase todas as combinações analisadas, com valores acima de zero, que índica um desempenho melhor que uma previsão aleatória. Vale notar que o melhor desempenho do WRF ocorreu com os esquemas de MPL e G3D, indicando valores de 0,5817 e 0,5319 em ambos os pontos. A análise também evidenciou que o pior resultado foi indicado pelos esquemas paramétricos de MPT e KF, com valores de 0 e $-0,0836$ o que significa que os esquemas são inadequado para previsão nesta região, apesar de terem apresentados uma boa estatística.

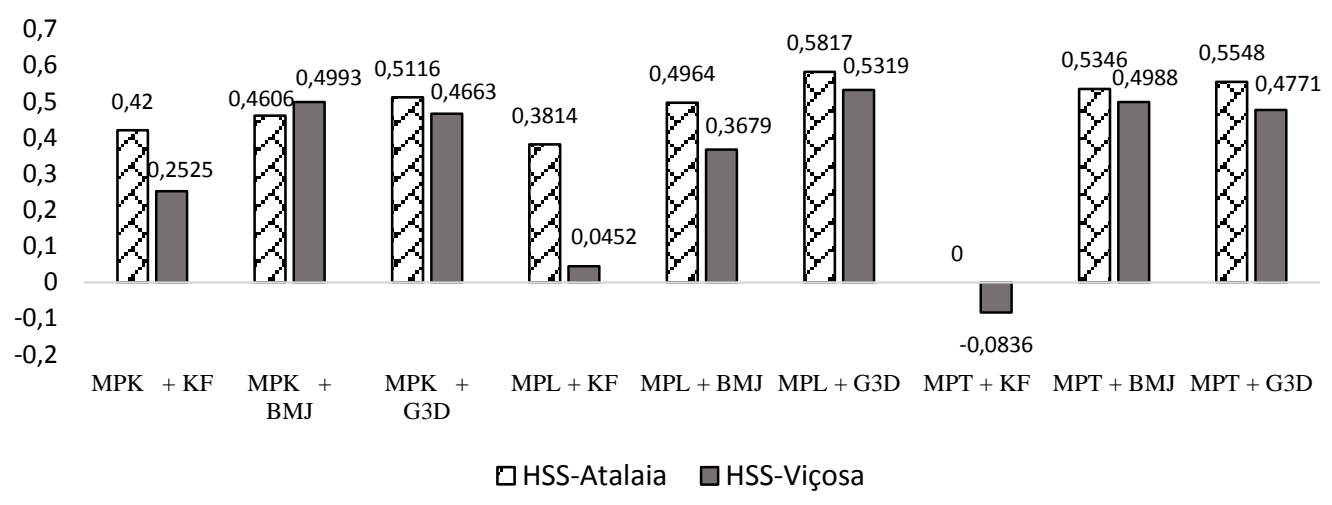

Figura 4: Índice de Habilidade (HSS) do modelo WRF para as combinações testadas

Os resultados obtidos no presente artigo possibilitará futuramente um possível acoplamento do tipo off-line entre o modelo WRF e um modelo hidrológico, no propósito de prever eventos extremos a curtíssimo prazo como cheias e inundações na bacia do rio Paraíba do Meio. Utilizando ideia semelhante Calvetti (2011) encontrou resultados satisfatórios nas previsões de cheias na bacia do Alto Iguaçu, no estado do Paraná, utilizando o WRF e o modelo TopModel. 


\section{CONSIDERAÇÕES FINAIS}

Com base nos resultados concluirmos que o modelo de mesoescala WRF-ARW apresenta uma tendência em subestimar as precipitações pluviométricas a curtíssmo prazo na bacia do rio Paraíba do Meio. A maioria das rodadas previstas apresentaram boas correlações, com intensidades não muito distantes das observadas. A topografia, a cobertura vegetal e o uso e ocupação do solo parecem ter influência os esquemas físicos. A combinação do esquema de microfísica de Thompson com a convecção de Kain-Fritsch, apresentou a melhor estatística entre as adaptações realizadas no estudo, contudo, o índice de HSS mostrou que o modelo WRF-ARW usando os esquemas de MPL e G3D oferecem uma melhor habilidade na estimativa de chuva para região da bacia do rio Paraíba do Meio.

\section{REFERÊNCIAS}

ARGÜESO, DANIEL, JOSÉ M. HIDALGO-MUÑOZ, SONIA R. GÁMIZ-FORTIS, MARÍA JESÚS ESTEBAN-PARRA, JIMY DUDHIA, YOLANDA CASTRO-DÍEZ, 2011: Evaluation of WRF Parameterizations for Climate Studies over Southern Spain Using a Multistep Regionalization. J. Climate, 24, 5633-5651.

BARNSTON, A. G., 1992: Correspondence among the correlations, RMSE, and Heidke forecast verification measures; refinement of the Heidke score. Wea. Forecasting,7, 699-709.

BETTS, A. K.; M. J. MILLER, 1986: A new convective adjustment scheme. Part II: Single column tests using GATE wave, BOMEX, ATEX and Arctic air-mass data sets. Quart. J. Roy. Meteor. Soc., 112, 693-709.

CALLEGARI-JACQUES, SIDIA M. Bioestatística: princípios e aplicações. Porto Alegre: Artemed, 2003. 255p.

CALVETTI, L. Previsão hidrológica probabilística na Bacia do Alto Iguaçu-PR com os modelos WRF e TopModel. 141 laudas. Tese (Doutorado em Ciências). Instituto de Astronomia, Geofísica e Ciências Atmosféricas da Universidade de São Paulo. São Paulo, 2011.

CHEN, F.; DUDHIA, J. Coupling an advanced land-surface/ hydrology model with the Penn State/ NCAR MM5 modeling system. Part I: Model description and implementation. Mon. Wea. Rev., v. 129, p. 569-585, 2001.

CONNER, MARK D., GRANT, W. PETTY, 1998: Validation and Intercomparison of SSM/I RainRate Retrieval Methods over the Continental United States. J. Appl. Meteor., 37, 679-700.

DOSWELL, C. A., III, R. DAVIES-JONES, AND D. L. KELLER, 1990: On summary measures of skill in rare event forecasting based on contingency tables. Wea. Forecasting, 5, 576-585.

DYER, A. J.; HICKS, B. B. Flux-gradient relationships in the constant flux layer, Quart. J. Roy. Meteor. Soc., v. 96, p. 715-721, 1970.

GRELL, G. A.; D. DEVENYI, 2002: A generalized approach to parameterizing convection combining ensemble and data assimilation techniques. Geophys. Res. Lett. 29, 1693-1696.

JANJIĆ, Z. I., 1994: The step-mountain Eta coordinate model: Further developments of the convection closure schemes. Mon. Wea. Rev.,122,927-945.

KAIN, J. S., AND J. M. FRITSCH, 1993: The role of the convective "trigger function"in numerical prediction of mesoscale convective systems. Meteor. Atmos. Phys.,49,93-106.

KESSLER, E., 1969: On the Distribution and Continuity of Water Substance in Atmospheric Circulation. Meteor. Monogr., No. 32, Amer. Meteor. Soc., 84 pp. 
LEE, R. R., AND J. E. PASSNER, 1993: The development and verification of TIPS: An expert system to forecast thunderstorm occurrence. Wea. Forecasting,8, 271-280.

LIN, Y. L.; FARLEY, R. D.; ORVILLE, H. D. Bulk parameterization of the snow field in a cloud model. Journal of Climate Appl. Meteorol., v. 22, p. 1065-1092, 1983.

LIRA, S. A. Análise de Correlação: Abordagem Teórica e de Construção dos Coeficientes com Aplicações. 209 Folhas. Dissertação (Mestrado em Métodos Numéricos em Engenharia dos Setores de Ciências Exatas). Universidade Federal do Paraná. CURITIBA, 2004.

MACEDO, L. R.; HENKES, A. F.; YAMASAKI, Y. Modelo WRF Na Previsão de um Evento Severo no Rio Grande do Sul. In: IV Encontro Sul-Brasileiro de Meteorologia, 2011, Pelotas. IV Encontro Sul-Brasileiro de Meteorologia, 2011. v. 4.

MLAWER, E. J., TAUBMAN, S. J., BROWN, P. D., IACONO, M. J. e CLOUGH, S. A. Radiative transfer for inhomogeneous atmosphere: RRTM, a validated correlated-k model for the longwave. J. Geophys. Res., 102 (D14), p. 16663-16682, 1997.

MOLLMANN JUNIOR, R, A.; SILVA JUNIOR. R. S.; GOMES, H. B.; MOURA, M. A. L. Estudo da Dispersão de Monóxido de Carbono (CO) Emitido Através da Queima de Cana-De-Açúcar, no Estado De Alagoas. Revista Brasileira de Geografia Física. V. 06 N. 03, 488-499, 2013.

MORIASI, D.N., ARNOLD, J.G., VAN LIEW, M.W., BINGNER, R.L., HARMEL, R.D., VEITH, T.L. Model evaluation guidelines for systematic quantification of accuracy in watershed simulations. American Society of Agricultural and Biological Engineers 50, 885-900. 2007.

OLIVEIRA, A. C. Implementação do Modelo Atmosférico WRF Acoplado Com o Modelo Hidrológico Topmodel Para a Bacia de União da Bitória. 112 folhas. Dissertação (Mestrado em Engenharia de Recursos Hídricos e Ambiental). Universidade Federal do Paraná. Curitiba, 2006.

PAULSON, C. A. The mathematical representation of wind speed and temperature profiles in the unstable atmospheric surface layer. J. Appl. Meteorol., v. 9, p. 857-861, 1970.

PLEIM JE. A combined local and nonlocal closure model for the atmospheric boundary layer. Part II: application and evaluation in a mesoscale meteorological model. J Appl Meteorol Clim 46, 2007, 1396-1409.

RAMOS, D. N. S.; LYRA, R. F. F.; SILVA JÚNIOR, R. S. S. Previsão do Vento Utilizando o Modelo Atmosférico WRF Para o Estado. Revista Brasileira de Meteorologia, v.28, n.2, 163 - 172, 2013.

SKAMAROCK, W. C.; KLEMP, J. B.; DUDHIA, J.; GILL, D. O.; BARKER, D. M.; WANG, W.; POWERS, J. G. A Description of the Advanced Research WRF Version 2. NCAR/Tech. Notes, 2005.

SKAMAROCK, W. C.; KLEMP, J. B.; DUDHIA, J.; GILL, D. O.; BARKER, D. M.; DUDA, M. G.; HUANG, X. -Y.; WANG, W.; POWERS, J. G. A description of the Advanced Research WRF Version 3. National Center for Atmospheric Research (NCAR). Boulder, Colorado (EUA), p. 125.2008.

THOMPSON, GREGORY, ROY M. RASMUSSEN, KEVIN MANNING, 2004: Explicit Forecasts of Winter Precipitation Using an Improved Bulk Microphysics Scheme. Part I: Description and Sensitivity Analysis. Mon. Wea. Rev., 132, 519-542.

WEBB, E. K. Profile relationships: The log-linear range, and extension to strong stability. Q. J. Roy. Meteor. Soc., v. 96, p. 67-90, 1970. 
WILKS, D. S.: Statistical methods in the atmospheric sciences. Academic Press, 467 p., 1995.

YUAN, HUILING, CHUNGU LU, JOHN A. MCGINLEY, PAUL J. SCHULTZ, BRIAN D. JAMISON, LINDA WHARTON, CHRISTOPHER J. ANDERSON, 2009: Evaluation of Short-Range Quantitative Precipitation Forecasts from a Time-Lagged Multimodel Ensemble. Wea. Forecasting, 24, 1838. 